\title{
Short-term retention of pictures and words as a function of type of distraction and length of delay interval
}

\author{
JAMES W. PELLEGRINO, ALEXANDER W. SIEGEL, and MEENA DHAWAN \\ University of Pittsburgh, Pittsburgh, Pennsylvania 15260
}

\begin{abstract}
Picture and word triads were tested in a Brown-Peterson short-term retention task at varying delay intervals $(3,10$, or $30 \mathrm{sec})$ and under acoustic and simultaneous acoustic and visual distraction. Pictures were superior to words at all delay intervals under single acoustic distraction. Dual distraction consistently reduced picture retention while simultaneously facilitating word retention. The results were interpreted in terms of the dual coding hypothesis with modality-specific interference effects in the visual and acoustic processing systems. The differential effects of dual distraction were related to the introduction of visual interference and differential levels of functional acoustic interference across dual and single distraction tasks. The latter was supported by a constant $2 / 1$ ratio in the backward counting rates of the acoustic vs. dual distraction tasks. The results further suggest that retention may not depend on total processing load of the distraction task, per se, but rather that processing load operates within modalities.
\end{abstract}

Recently, Pellegrino, Siegel, and Dhawan (1975) reported a series of experiments in which differential retention of pictures and words was demonstrated in a Brown-Peterson short-term retention task. Specifically. (1) retention of pictures was superior to retention of words under conditions of acoustic distraction (counting backwards), (2) pictures and words were equally well retained under conditions of visual distraction (solving an embedded figures task), but (3) simultaneous visual and acoustic distraction reduced retention of pictures while also producing a slight facilitation of word retention. These results were interpreted as supporting a dual encoding of pictures as contrasted with a single acoustic encoding for words (cf. Paivio, 1971) with modality-specific interference effects for each stimulus representation (e.g.. Brooks. 1968).

The present research was designed to further investigate the nature of the differential performance as a function of the length of the delay (interference) interval and the type of distraction. Previously, higher retention of pictures relative to words under acoustic distraction had shown after a 15 -sec delay interval and was attributed to a visual stimulus representation which was not subject to interference from the acoustic task. Superior retention of pictures might also be predicted at short retention intervals, while the

This research was supported by funds provided by the Learning Research and Development Center. University of Pittsburgh, which is supported in part by the National Institute of Education, U.S. Departnent of Health. Education, and Welfare. Reprint requests should be addressed to James W. Pellegrino, Department of Psychology. University of Pittsburgh, Pittsburgh. Pennsylvania 15260 . effect might decline over the course of longer intervals if the visual stimulus representation decays or cannot be maintained through rehearsal. Similarly, the detrimental effect of simultaneous visual and acoustic interference might be expected at short intervals with a convergence of the performance produced by single and dual distraction conditions at longer delay intervals. However, if the visual stimulus representation can be maintained for long intervals (possibly through rehearsal), then the superior retention of pictures under acoustic distraction and reduction in recall under dual distraction should occur at all delay intervals.

An additional concern in the present design was the relative effects of single and dual distraction on word recall. A facilitation in word recall under dual distraction might be due to a lower level of functional acoustic interference as a result of the overall processing demands of the simultaneous distraction task. In the present experiment, an attempt was made to specify the relationship of recall to the level of acoustic interference by monitoring the backward counting rate in both single and dual distraction conditions.

Previous research on short-term retention (e.g. Dillon \& Reid, 1969; Posner \& Rossman, 1965) has shown performance decrements for recall as a function of the processing demands of the distraction task. In the present design, the dual distraction task involves considerable processing load. However, recall of pictures and words across single and dual distraction may not be a function of processing difficulty, per se, but rather the source(s) and level(s) of the interference generated during the distraction 


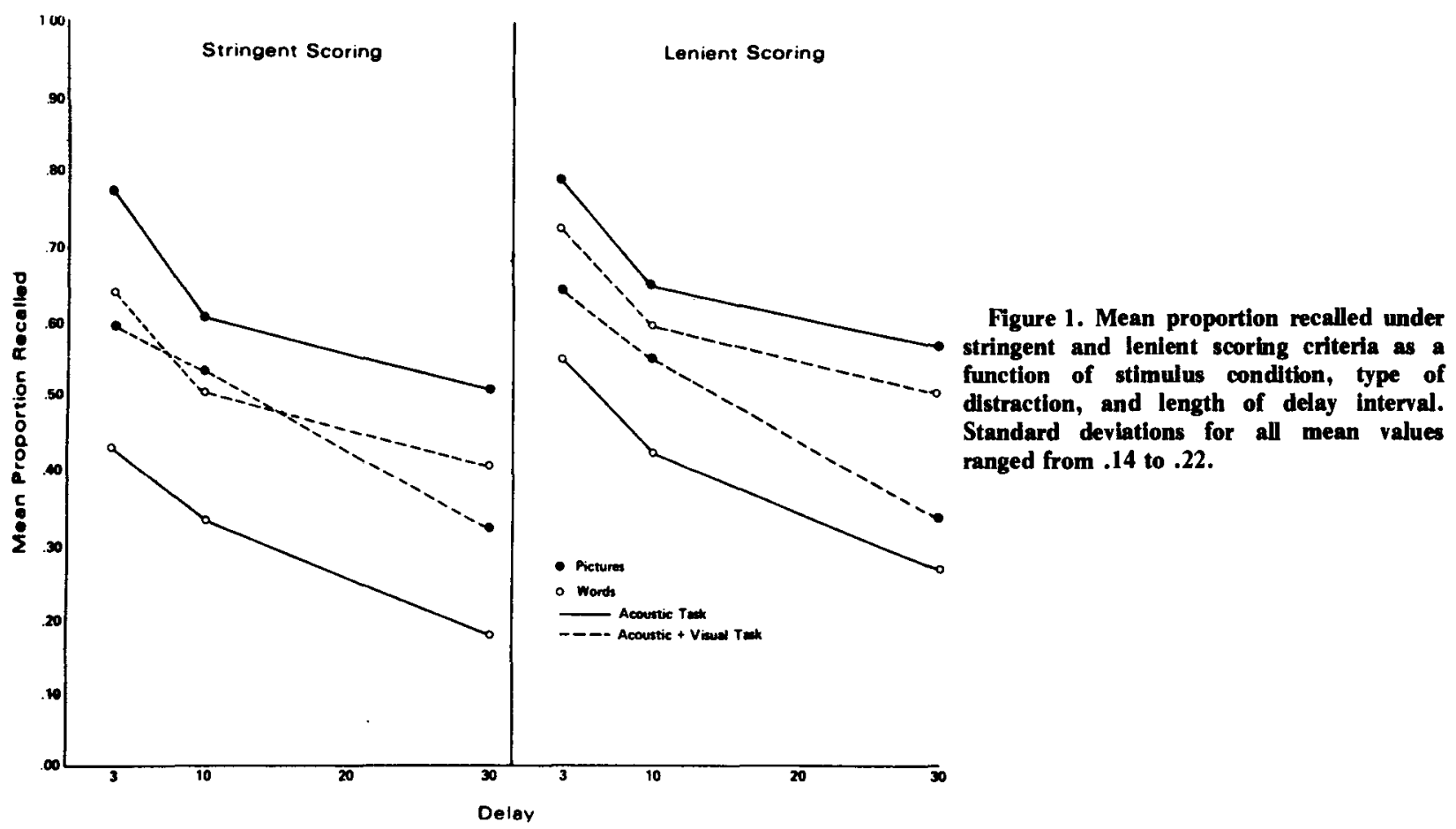

interval. In the case of pictures, dual distraction may involve a lower level of functional acoustic interference; but the accompanying introduction of visual distraction may produce interference with the rehearsal of information in both storage or processing systems. In the case of words, the introduction of visual distraction, while increasing overall processing load, may lower the level of acoustic interference, thereby facilitating overall performance. Such a differential pattern of distraction effects for pictures and words would lead to the conclusion that, although the processing demands of the distraction task are important in determining performance, this influence is restricted to the particular modality of stimulus representation.

\section{METHOD}

\section{Design and Procedure}

The present design was a 2 by 2 by 3 mixed factorial with one between-subjects factor, stimulus modality (pictures vs. words), and two within-subjects factors, type of distraction (acoustic vs. acoustic + visual) and delay interval $(3,10$, or $30 \mathrm{sec})$. All subjects received a 24 -trial sequence involving the presentation of 24 word or picture triads. Each trial consisted of a 2 -sec visual presentation of the stimuli, which were diagonally staggered on the slide, followed by 3,10 , or $30 \mathrm{sec}$ of the particular distraction task. On each trial, the subject was asked to orally recall each item and its position within the triad.

The acoustic distraction task involved backward counting in units of seven from a specitied three-digit number. The acoustic + visual distraction task involved simultaneous backward counting and working on a modified version of the Hidden Figures Test. (The latter consisted of trying to locate a nonlabelable shape in a matrix of confusing lines and outlining the shape in that matrix.)

The 24 trials were divided into four blocks of six trials each, with each type of distraction occurring in two different trial blocks.
Within a trial block, each delay interval occurred twice. All eight possible orderings of trial blocks were used across subjects, and each triad was used equally often for each combination of delay and distraction.

\section{Materials}

The 24 picture triads and their corresponding word triads were drawn from materials used by Pellegrino et al. (1975). These consisted of 72 black line drawings of highly familiar objects and animals. The corresponding verbal labels of these pictures were one or two syllables in length, with syllabic length varied within item triads. The pictorial stimuli were previously shown to be readily labelable by young children.

\section{Subjects}

The subjects were 48 University of Pittsburgh undergraduates participating to fultill a requirement for introductory psychology. All subjects were tested individually.

\section{RESULTS}

\section{Recall}

Recall performance was scored by two criteria. Under the stringent scoring criterion, items were considered correct only if recalled and assigned the correct position within the triad. The lenient scoring criterion disregarded the position assignment and considered items as correct if recalled at all. The results of both scoring criteria are shown in Figure 1. As can be seen in this figure, under the stringent scoring system recall of pictures $(.56)$ was significantly greater than that of words $(.41)[F(1,46)=25.75$, $\mathrm{p}<.001]$. However, the picture-word effect interacted with type of distraction $[F(1,46)=70.42$, $\mathrm{p}<.001]$. Tests of simple main effects showed picture recall (.63) to be superior to word recall $(.31)$ under acoustic distraction and no difference between the two 


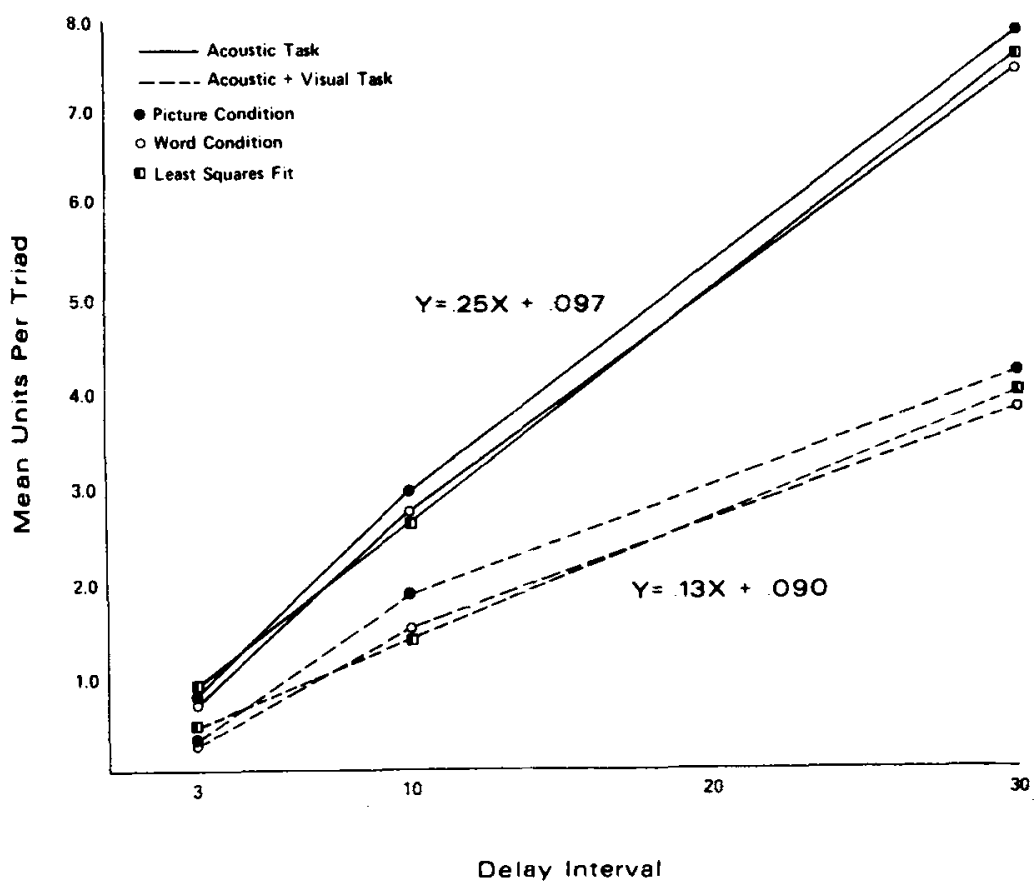

Figure 2. Backward counting rate expressed in terms of units of seven per triad. Shown as a function of stimulus condition, distraction condition, and length of delay interval. Also shown are least squares regression equations for each distraction condition pooled across stimulus conditions. In each distraction condition, standard deviations increased over delay intervals, with respective values of $.37,1.19$, and 3.29 for acoustic distraction and $.25, .60$, and 1.51 for acoustic + visual distraction. under acoustic + visual distraction (.48 vs. .51 ). Picture recall was significantly better under acoustic than under dual distraction, while word recall was significantly better under dual distraction! The only other significant effect was the drop in performance across delay intervals $[F(2,92)=56.44, p<.001]$, and this did not interact with the other two factors (ps $>.10$ ).

The analysis of performance under the lenient scoring criterion produced an almost identical pattern of results. The recall of pictures $(.59)$ was superior to that of words $[F(1,46)=8.01, p<.01]$. The picture-word difference again interacted with type of distraction $[F(1,46)=103.64, p<.001]$. Tests of simple main effects again showed pictures (.67) superior to words (.41) under acoustic distraction and the opposite effect under dual distraction (.51 vs. .61). Picture recall was significantly better under acoustic than under dual distraction, while just the opposite occurred for words. Again. recall declined over the delay intervals $[F(2,92)$ $=59.04 . \mathrm{p}<.001]$, and this did not interact with the other two factors (ps $>.10$ ).

An additional analysis was performed on recall gain in going from the stringent to the lenient scoring criterion. This analysis showed a greater gain for words $(.095)$ than for pictures $(.033)[\mathrm{F}(1,42)=$ 19.18. $\mathrm{p}<.001]$. The only other effect was a marginally significant interaction involving all three variables $[F(2,92)=3.14, p>.05]$. No other effect or interaction approached significance $(\mathrm{Fs}<1)$.

\section{Backward Counting}

In both the acoustic and acoustic + visual distraction tasks, the rate of backward counting was assessed in terms of the units of seven generated during the various delay intervals. Figure 2 presents the results of this analysis in terms of the units per triad. As can be seen in this figure, the rate was slower in the acoustic + visual (2.01) than in the acoustic (3.74) tasks $[\mathrm{F}(1,46)=129.53, \mathrm{p}<.001]$. The rates increased over delay intervals $[F(2,92)=304.31$, $\mathrm{p}<.001]$, with means of .54, 2.37, and 6.02 for 3,10 , and $30 \mathrm{sec}$. respectively, and the difference between the two distractor tasks increased over the delay intervals $[F(1,92)=78.88, p<.001]$. Figure 2 also shows that these differences in the rates of backward counting were constant across the different groups of subjects receiving word and picture stimuli. Also shown in Figure 2 are the equations for the least squares regression lines for the counting rates in the two distraction tasks. As is clear, the intercepts approximate zero and the slope of the function in the dual distraction task is approximately one-half that of the single acoustic distraction task.

\section{DISCUSSION}

The present results are similar to those reported by Pellegrino et al. (1975) obtained under conditions of acoustic distraction. The superiority of picture over word recall argues in favor of dual encoding of pictures (cf. Paivio, 1971), with the visual and acoustic (verbal) representations of the stimuli residing in separate processing or storage systems. The existence of such dual stimulus representations should facilitate performance if the primary effect of the backward counting task is to interfere with rehearsal in the acoustic storage system rather than affecting the visual storage system (Wells, 1972). It is also clear that the visual stimulus representation serves to facilitate performance at all delay intervals 
with no evidence of an interaction between picture-word retention and length of the delay interval. Thus the visual representation can be maintained for relatively long periods of time, similar to results reported by Parkinson (1972) for visually presented letters. However, it is not immediately clear whether this is due to slow rates of trace decay or maintenance of the trace through a form of rehearsal (e.g., Kroll \& Kellicutt, 1972).

There are two additional results which support the hypothesis of dual encoding of pictures. The first of these is the differential gain performance for word and picture recall from lenient to stringent scoring criteria. As Pellegrino et al. (1975) have argued, the positional or sequential order information for word triads may be a function of the temporal ordering of item entry into the acoustic store. Such order information may be maintained via rehearsal, but the backward counting task may disrupt the temporal order information while having less of an effect upon individual item retention (see also Bjork \& Healy, 1974). Thus, relatively large performance gains for word stimuli would be expected across the two scoring criteria. By contrast, if the pictures are visually represented, then this representation would contain both item and order information. If recall is from this visual store, then if an item is recalled, its position should also be correctly retained and low performance gains across criteria would be expected. That this occurred for pictures under both distraction conditions and at all delay intervals would indicate that the attempted recall of picture stimuli is primarily from the visual store (see also Salzberg, Parks, Kroll, \& Parkinson, 1971).

The second result which supports a dual coding position is the reliable performance decrement for pictures when simultaneous visual and acoustic distraction is compared to acoustic distraction alone. Such a result would be predicted, since both encodings of the stimulus were subjected to interfering distraction tasks. The relative consistency of the dual distraction effect for pictures also argues that this interference effect is operating equally at all delay intervals. Again, these results are consistent with those reported by Pellegrino et al. (1975).

Of particular concern in this study was the possibility of differential effects of the dual distraction task on the retention of pictures and words. Such a differential pattern was clearly evident at all delay intervals. There are at least three possible predictions that could have been made about the general effects of dual distraction compared to single acoustic distraction. One such prediction might be that the two distraction conditions are equivalent and therefore picture (or word)retention should not differ across tasks. The present results plus those reported by Pellegrino et al. (1975) clearly violate this prediction. A second prediction might be that the simultaneous performance of the two tasks produces more total interference than the acoustic task by itself. Thus both picture and word retention should drop under dual distraction-this did not occur for word recall. The third prediction is based upon modality-specific interference effects. Such a position would predict picture performance to drop under dual distraction while word recall could remain stable. However, word recall did not remain stable; rather, it significantly increased under the dual distraction task. The latter finding introduces the possibility of different levels of acoustic interference across the single and dual distraction tasks.

The analysis of the backward counting rates clearly showed that the introduction of the visual distraction task reduced the rate of counting to one-half that for when the acoustic task was presented alone. A reduced counting rate can be readily interpreted as indicating that the subjects were time-sharing their processing effort across the two tasks, thereby reducing the level of functional acoustic interference in the dual distraction task to one-half that obtained under single acoustic distraction. Such a reduction in acoustic interference would be expected to increase recall performance from the acoustic store, and such a result was obtained for the word stimuli across all delay intervals.

A similar facilitation in performance would be expected for the acoustic (verbal) representation of the picture stimuli. However, while the level of functional acoustic interference is less under dual distraction, there is the simultaneous introduction of visual interference which was not present under the acoustic distraction alone. Thus the facilitation in retention for the acoustic store is pitted against interference in the visual store, thereby decrementing overall performance on pictures.

As noted in the introduction, this differential pattern of interference effects seems to argue that processing demands of the interference task determine performance levels, but this relationship operates within particular modalities. Previous investigations of processing demands (e.g., Kroll \& Kellicutt, 1972; Posner \& Rossman, 1965) have dealt primarily with acoustically encoded stimuli and distraction tasks which produced interference primarily in the acoustic modality. The present dual distraction task is extremely difficult and may place more of a processing load upon the system than either task alone. However, the effects of the dual task are differential on picture and word recall due to the amount of rehearsal-preventive interference generated within each processing system. Facilitation for words is due to less total interference in the acoustic processing system. The corresponding decrement for pictures is due to the increased visual interference which counteracts the decrement in acoustic interference. Thus it appears that processing 
demands, per se, are not the determinant of performance, but rather that retention depends upon the locus of the processing effort.

\section{REFERENCES}

Bjork, E. L., \& Healy, A. F. Short-term order and item retention. Journal of Verbal Learning and Verbal Behavior, 1974, 13, 80-97.

Brooks, L. R. Spatial and verbal components of the act of recall. Canadian Journal of Psychology, 1968, 22, 349-368.

Dillon, R. F., \& Reid, L. S. Short-term memory as a function of information processing during the retention interval. Journal of Experimental Psychology, 1969, 81, 261-269.

Kroll, N. E., \& KellicutT, M. H. Short-term recall as a function of covert rehearsal and intervening task. Journal of Verbal Learning and Verbal Behavior, 1972, 11, 196-204.

Parvio, A. Imagery and verbal processes. New York: Holt, Rinehart, \& Winston, 1971.
Parkinson, S. R. Short-term memory while shadowing: Multiple item recall of visually and of aurally presented letters. Journal of Experimental Psychology, 1972, 92, 256-265.

Pellegrino, J. W., Siegel, A. W., \& Dhawan, M. Shortterm retention of pictures and words: Evidence for dual coding systems. Journal of Experimental Psychology: Human Learning and Memory, 1975, 104, 95-102.

Posner, M. I., \& Rossman, E. Effect of size and location of informational transforms upon short-term retention. Joumal of Experimental Psychology, 1965, 70, 496-505.

Salzberg, P. M., Parks, T. E., Kroll, N. E., \& Parkinson, S. R. Retroactive effects of phonemic similarity on short-term recall of visual and auditory stimuli. Journal of Experimental Psychology, 1971, 91, 43-46.

Wells, J. E. Encoding and memory for visual and pictorial stimuli. Quarterly Joumal of Experimental Psychology, 1972, 24, $242-252$.

(Received for publication January 27, 1975; accepted March 10, 1975.) 